\title{
Regolarità alla frontiera di soluzioni di equazioni ellittiche (*).
}

\author{
Memoria di Carlo Pucci (a Genova)
}

Sunto. - Si studia la hölderianità in prossimita della frontiera delle soluzioni delle equazioni uniformemente ellittiche del secondo ordine.

Summary. - We consider uniform elliptic equations of the second order in a domain with the exterior cone property and we investigate the Holder exponent and coefficient of the solutions near the boundary. We prove that both are independent by the continuity of the differential operator wit respect to the variables.

Si studia la regolarità alla frontiera delle soluzioni del problema di Dirichlat per le equazioni uniformemente ellittiche del secondo ordine:

$$
\text { (*) } F\left(x, u, u_{i}, u_{i j}\right)=0, x \in \Omega \text {, }
$$

ove $\boldsymbol{\Omega}$ è un aperto limitato con la proprietà esterna di cono. Sotto opportune ipotesi si prova che se $u$ è una soluzione della equazione ed è holde. riana con esponente $\lambda$ su $\partial \Omega$, risulta

$$
\left|u(x)-u\left(x^{0}\right)\right| \leq H\left|x-x^{0}\right|^{2} \quad \text { con } \quad x \in \Omega, x^{0} \in \partial \Omega
$$

ove $H$ dipende solo da $F, \Omega$ e dalla holderianità di $u$ su $\partial \Omega$.

Vi sono numerosi studi sulla regolarita alla frontiera delle soluzioni di equazioni di tipo ellittico. Ricordo in breve che LeBEsGue [8] ha costruito il primo esempio di aperto $\Omega$ relativamente al quale la «soluzione» del problema di DIRICHLen per l'equazione di Laplace può non essere continua in punti della frontiera pur essendo continua la funzione assegnata sulla frontiera; Wrener [20] stabilì le condizioni necessarie e sufficienti relative a $\partial \Omega$ affinchè il problema di DIRICHLET per l'equazione di LAPlace am. metta una soluzione continua in $\overline{\mathbf{Q}}$ quando la funzione assegnata su $\partial \mathrm{Q} \dot{\mathrm{O}}$ continua. I risultati di WIENER sono stati estesi alle equazioni lineari ellittiche del secondo ordine con coefficienti differenziabili da Püscher [17], Tautz [19], Oleinik [14], Herné [6] e sono stati ultimamente estesi alla

(*) Lavoro eseguito nell' ambito del gruppo, di ricerca n. 23 del Comitato per la mate. matica del CNR nell'anno accademico 1963-64. I risultati di questo lavoro sono stati in parte precedentemente esposti in [16], con dimostrazione parzialmente diversa. 
equazione variazionale

$$
(* *) \Sigma \frac{\partial}{\partial x_{i}}\left[a_{i j}(x) \frac{\partial u}{\partial x_{i}}\right]=0, \quad x \in \Omega
$$

da Ltttman, Stampacohia e Weinberger [9] nella sola ipotesi che i coefficienti $a_{i j}$ siano misurabili in $\boldsymbol{Q}$ e l'equazione uniformemente ellittica.

La ricerca di Litmman, Stampacchia, e Weinberger può anche essere considerata come un completamento dei risultati di DE GIORGI [4], NASH [12], Moser [11], relativi alla maggiorazione, all'interno di $\mathbf{\Omega}$, del coefficiente di HöLoEr delle soluzioni della $(* *)$ indipendentemente dal modulo di continuità dei coefficienti $a_{i j}$.

Analoghi risultati per equazioni in forma non autoaggiunta erano finora noti solo nel caso di due dimensioni (Nirenberg [13], Bers Nurenberg [2], Fins [b]); con la presente ricerca viene stabilita la hölderianita alla frontiera della soluzione indipendentemente dal modulo di continuità dell'operatore rispetto ad $x$.

$S i$ noti che le ipotesi riguardanti $\partial \Omega$ sono, in questo studio, piủ restrittive di quelle di WIENER e ciò è inevitabile, come è mostrato con un esempio, richiedendosi la hölderianità della soluzione alla frontiera e non la sola continuità.

Condizioni di regolarità (maggiore della continuità) alla frontiera per soluzioni di equazioni di tipo ellittico sono state considarate da vari Autori. Kellog [7] ha provato che in opportune ipotesi su $\partial \Omega$ la soluzione del problema di DiRICHLeT per l'equazione di Laplace è di classe $C^{(n, \lambda)}$. Questi risultati sono stati estesi alle equazioni lineari di tipo ellittico del secondo ordine con coefficienti hölderiani da Schauder [18], Caocioppolr [3], Mrranda [10]. Risultati più generali sono stati ottenuti da AGMon, Douglis, Nirenberg [1] relativamente al comportamento alla frontiera di soluzioni di equazioni lineari di tipo ellittico di ordine qualsiasi.

Le ipotesi relative a $\partial \Omega$ considerate nelle ricerche menzionate per provare la hölderianità alla frontiera della soluzione, anche nel caso della equazione di LAPLACE, sono più restrittive di quelle considerate nel presente studio; inoltre, diversamente dalle precedenti ricerche, qua non si suppone la limitatezza in $\Omega$ di $F$; e delle derivate $F_{u}, F_{u_{i}}$; esse possono divenire infinite di ordine opportuno per $x$ tendente a $\partial \Omega$.

Notiamo che sulla base di questi risultati si possono ottenere nuovi teoremi di esistenza per il problema di DIRICHLET per equazioni lineari ellittiche con coefficienti hölderiani internamente ad $\Omega$ ed infiniti di ordine opportuno per $x$ tendente a $\partial Q$. 
In una mia precedente nota [15] sono stabilite condizioni di hölderianita della soluzione di una equazione ellittica in un punto della frontiera dello insieme di definizione; si tratta di un risultato non completamente contenuto nei teoremi qua stabiliti ed ottenuto con una dimostrazione abbastanza diversa da quella qua esposta.

Il procedimento dimostrativo seguito in questa ricerca è abbastanza complicato; si basa sul principio di massimo e su considerazioni analitiche del tutto elementari.

\section{1. - Ipotesi e proprietà riguardanti $\mathbf{Q}$.}

Supponiamo sempre che $\mathbf{\Omega}$ sia un insieme aperto, connesso, limitato dello spazio euclideo ad $m$ dimensioni.

Diciamo che $\Omega$ è di classe $H_{\beta, \delta}$, con $\beta$ e $\delta$ costanti positive $\beta<\frac{\pi}{2}$, se per ogni punto $x^{0} \in \partial \Omega$ vi è un cono circolare retto esterno ad $\Omega$, di vertice $x^{0}$, apertura $\frac{\pi}{2}-\beta$, altezza $\delta$.

Osserviamo che ogni aperto $\Omega$ convesso appartiene a tutte le classi $H_{\beta, 8}$ $\operatorname{con} \beta$ e $\delta$ positivi, $\beta<\frac{\pi}{2} ;$ ogni aperto $\Omega$, dotato in ogni punto $2 \Omega$ di iperpiano tangente variabile con continuità, appartiene ad $H_{\varepsilon, \delta}$ con $0<\varepsilon<\frac{\pi}{2} e$ per il resto arbitrario e $\delta$ opportuno, dipendente da $\varepsilon$ ed $\Omega$; ogni aperto $\Omega$ poliedrico, cioè con frontierà contenuta in numero finito di iperpiani, appartiene ad una qualche classe $H_{\beta, \delta}$ con $\beta$ e $\delta$ opportuni.

Se $\mathbf{\Omega} \in H_{\beta, \delta}$ per ogni punto $x^{0} \in \partial \Omega$ esiste almeno un versore $n^{0}$ tale che se $x \in \mathrm{Q},\left|x-x^{0}\right|<\delta$, l' angolo $\left[x-x^{0}, n^{0}\right]$ formato fra $i$ vettori $x-x^{0}$. ed $n^{0}$ è minore di $\frac{\pi}{2}+\beta$. Ad esempio si può assumere come versore $n^{0}$ quello penetrante in $\Omega$ ed avente la stessa direzione dell'asse del cono, esterno ad $\Omega$, di vertice $x^{0}$, apertura $\frac{\pi}{2}-\beta$ ed altezza $\delta$.

Si suppone da qui in avanti $\Omega \in H_{\beta, \delta}$ e si suppone definito il versore $n^{0}=n\left(x^{0}\right)$ al variare di $x^{0}$ su $\partial \mathrm{Q}$, con le proprieta sopra indicate.

Indichiamo con $d(x)$ la distanza di un punto $x$ da $\partial \Omega$.

Fissato un punto $x^{0} \in \partial \Omega$ e una costante $t, 0<t<1$, poniamo

$$
\begin{aligned}
& T\left(x^{0}, t\right) \equiv\left\{x ; x \in \Omega, \quad\left|x-x^{0}\right|<\delta, t\left|x-x^{0}\right|<d(x)\right\} \\
& \gamma_{t}=\sup _{\substack{x^{0} \in \delta \Omega \\
x \in T\left(x^{0}, i\right)}} \text { angolo }\left[x-x^{0}, n\left(x^{0}\right)\right] .
\end{aligned}
$$


Osserviamo che $\gamma_{t}$ decresce al crescere di $t$ in $(0,1)$ e pertanto possiamo definire

$$
\gamma_{1}=\lim _{t \rightarrow 1^{-}} \gamma_{t}
$$

Proviamo ohe

$$
\gamma_{1} \leq \beta
$$

Si ha infatti che se $\left|x-x^{0}\right|<\delta$ e l'angolo fra i vettori $x-x^{0}$ ed $n\left(x^{0}\right)$ è maggiore di $\beta$, la distanza di $x$ dal cono con asse parallelo ad $n\left(x^{0}\right)$ vertice in $x^{0}$, apertura $\frac{\pi}{2}-\beta$, altezza $\delta$, è minore di $\left|x-x^{0}\right|$ e pertanto $d(x)$ è minore di $\left|x-x^{0}\right|$ e quindi $x$ non appartiene a $T\left(x^{0}, t\right)$ per $t$ sufficientemente prossimo ad 1.

Proviamo inoltre che

$$
\gamma_{t} \leq \beta+\arccos t, \quad 0 \leq t \leq 1
$$

Basta provare che nel caso $\gamma_{t}>\beta$ si ha:

$$
t \leq \cos \left(\gamma_{t}-\beta\right)
$$

Per la definizione di $\gamma_{t}$, fissato $\varepsilon, 0<\varepsilon<\gamma_{t}-\beta$, esistono due punti $x, x^{0}$, con $x^{0} \in \partial \Omega, x \in \Omega,\left|x-x^{0}\right|<\delta,\left|x-x^{0}\right| t=d(x)$, e tali che l'angolo $\left[x-x_{0}, n\left(x^{0}\right)\right]>\gamma_{t}-\varepsilon$. Indicato con $\bar{x}$ il punto dell' insieme

$$
\left\{x ;\left|x-x^{0}\right| \leq \delta,\left[x-x^{0}, n\left(x^{0}\right)\right] \geq \frac{\pi}{2}+\beta\right\}
$$

più prossimo ad $x$, essendo $\Omega \in H_{\beta, \delta}$ risulta $|x-\bar{x}|>d(x)$ e quindi $|x-\bar{x}|>\left|x-x^{0}\right| t$.

Il triangolo $x, x^{0}, \bar{x}$ è rettangolo in $\bar{x}$ e l'angolo in $x^{0} \dot{\mathrm{e}}$ minore di $\frac{\pi}{2}+\beta-\gamma_{t}+\varepsilon$.

Si ha pertanto $|x-\bar{x}|=\left|x-x^{0}\right| \operatorname{sen} \widetilde{x^{0}}<\left|x-x^{0}\right| \cos \left(\gamma_{t}-\beta-\varepsilon\right)$ e quindi $t<\cos \left(\gamma_{t}-\beta-\varepsilon\right)$. Per l'arbitrarietà di $\varepsilon$ si ottiene la (5). 
2. Ipotesi su $F$. - Sia $F\left(x, u, u_{i}, u_{i j}\right)$ nna funzione reale definita nell' insieme $E$ dei punti $\left(x, u, u_{i}, u_{i j}\right)$ con $x \in \Omega$ ed $u, u_{i}, u_{i j}$ reali qualsiasi $(i, j=1, \ldots, m)$. Supponiamo the in $E$ la funzione $F$ sia dotata di derivate parziali rispetto ad $u, u_{i}, u_{i j}$ e vi siano delle costanti positive $x, \lambda, t, K$, con $\alpha, \lambda, t$ minori di 1 tali che

$$
\alpha|\mu|^{2} \leq \sum_{i, j}^{1, m} \frac{\partial F}{\partial u_{i j}^{i}} \mu_{i} \mu_{j} \leq|\mu|^{2} \text { in } E
$$

$$
d^{2}(x) \sum_{i=1}^{m}\left(\frac{\partial F}{\partial u_{i}}\right)^{2} \leq K^{2}, \text { in } E
$$$$
d^{2-\lambda}(x)\left|\frac{\partial F}{\partial u}\right| \leq K, \text { in } E,
$$

$$
d(x) n\left(x^{0}\right) \times\left(\frac{\partial F}{\partial u_{1}}, \ldots, \frac{\partial F}{\partial u_{m}}\right)<\frac{\alpha}{8}(1-\lambda) \text { per } x^{0} \in \partial \Omega, x \in T\left(x^{0}, \frac{1}{2}\right)
$$

l'ultima relazione dovendo essere soddisfatta per $u, u_{i}, u_{i j}$ reali qualsiasi.

Notiamo che le (8), (9), (10) sono soddisfatte qualora $F$ abbia derivate parziali rispetto ad $u$ ed $u_{i}$ limitate in $E$ (previa una opportuna scelta di $K$ e $\delta$ ).

Supponiamo inoltre che sia finito

$$
\sup _{x \in \mathbb{Q}}|F(x, 0,0,0)| d^{2-\lambda}(x)=\mathscr{F}
$$

Osserviamo che le ipotesi su $F$ sono invarianti rispetto a trasformazioni ortonormali di coordinate:

$$
\xi_{h}=\sum_{i=1}^{m} \varepsilon_{i, h} x_{i}, \text { con } \delta_{i, j}=\sum_{h, k}^{1, m} \varepsilon_{i, h} \varepsilon_{j, k}
$$

Infatti, posto

$$
v(\xi)=u[x(\xi)], \quad G\left(\xi, v, v_{h}, v_{h k}\right)=F\left(x, u, u_{i}, u_{i j}\right), \quad v\left(\xi^{\circ}\right)=n\left(x^{0}\right)
$$

si verifica facilmente

$$
\begin{gathered}
\sum_{h=1}^{m}\left(\frac{\partial G}{\partial v_{h}}\right)^{2}=\sum_{i=1}^{m}\left(\frac{\partial F}{\partial u_{l}}\right)^{2}, \\
\vee\left(\xi^{0}\right) \times\left(\frac{\partial G}{\partial v_{1}}, . ., \frac{\partial G}{\partial v_{m}}\right)=n\left(x^{0}\right) \times\left(\frac{\partial F}{\partial u_{1}}, \ldots, \frac{\partial F}{\partial u_{m}}\right),
\end{gathered}
$$


316 C. PUCCI : Regolarità alla frontiera di soluzioni di equazioni ellittiche

$$
\sum_{h_{i}, k}^{1, m} \frac{\partial G}{\partial v_{h k}} \lambda_{h} \lambda_{k}=\sum_{i, j}^{1, m} \frac{\partial F}{\partial u_{i j}} \mu_{i} \mu_{j} \text { con } \lambda_{h}=\sum_{i=1}^{m} \varepsilon_{i h} \mu_{i}
$$

\section{Enunciati.}

Sia $\Omega$ di classe $H_{\beta, \delta} \lambda$ una costante con $0<\lambda<1$, ed u una funzione di classe $C^{(2)}$ in $\Omega, C^{(0)}$ in $\overline{\mathbf{\Omega}}$, soluzione della equazione.

$$
F\left[x, u(x), u_{i}(x), u_{i j}(x)\right]=0 \quad x \in \mathbf{Q},
$$

verificante la condizione

$$
\left|u(x)-u\left(x^{0}\right)\right| \leq h\left|x-x^{0}\right|^{2} \text { per } x, x^{0} \in \partial \Omega
$$

Nelle ipotesi su $F$ indicate esiste una costante positiva $\beta_{0}$, dipendente solamente da $\alpha, \lambda, K, m$, tale che se $\beta \leq \beta_{0}$ risulta

$$
\left.\left|u(x)-u\left(x^{0}\right)\right| \leq \tau\left|h+\mathscr{F}+\max _{x \in \overline{0}}\right| u(x) \mid\right\}\left|x-x^{0}\right|^{\lambda} \text { per } x^{0} \in \partial \Omega, x \in \Omega,
$$

ove $\tau \grave{e}$ una costante dipendente solo $d a \alpha, \lambda, k, m, \delta$.

Notiamo dae immediati corollari del teorema precedente riferiti per semplicità ad equazioni lineari. Nél primo si introduce l'ipotesi restrittiva della limitatezza dei coefficienti; nel secondo si considera un particolare tipo di singolarita dei coefficienti comprendendo nella trattazione le equazioni generalizzate della teoria del potenziale a simmetria assiale. condizioni

II. - Siano $a_{i j}, b_{i}, c, f$ funzioni reali definite in $\Omega$ verificanti le

$$
\begin{aligned}
& \left|a_{i j}\right|,\left|b_{i}{ }^{\prime},\right| c \mid \leq M \quad \text { in } \Omega, \\
& \sum_{i, j}^{1, m} a_{i j} \mu_{i} \mu_{j} \geq \alpha|\mu|^{2} \quad \text { in } \Omega,
\end{aligned}
$$

con $\alpha$ e $M$ costanti positive fissate, $\alpha<1$. Sia u una funzione di classe $C^{(2)}$ in $\Omega, C^{(0)}$ in $\Omega$, soluzione della equazione

$$
\sum_{i, j}^{1, m} a_{i j} u_{i j}+\sum_{i=1}^{m} b_{i} u_{i}+c u-f=0 \text { in } \quad \mathrm{Q}
$$

e verificante la condizione (13) ove $\lambda$ è una costante, $0<\lambda<1$. Esiste una 
costante positiva $\beta_{0}$, dipendente solo da $\alpha, \lambda, M, m$ tale che se $Q \in H_{\beta, \delta}$ con $\beta \leq \beta_{0}$ si ha

$$
\begin{array}{r}
\left|u(x)-u\left(x^{0}\right)\right| \leq \tau\left\{h+\max _{x \in \bar{Q}}|u(x)|+\sup _{x \in \Omega} d^{2-\lambda}(x)|f(x)|\right\}\left|x-x^{0}\right|^{\lambda} \\
\text { per } x^{0} \in \partial \Omega, \quad x \in \Omega
\end{array}
$$

con $\tau$ dipendente solo $d a \alpha, M, \lambda, \delta, m$.

III Supponiamo $\Omega$ contenuto nel semispazio $x_{m}>0$ e la parte della frontiera $d i \Omega$ non contenuta nell' iperpiano $x_{m}=0$ sia dotata in ogni suo punto di iperpiano tangente variabile con continuità uniforme.

Siano $a_{i j}, b_{i}, c, f$ funzioni reali definite in $\Omega$ verificanti le (15), (16) ed inoltre

$$
b_{m}<\frac{\alpha}{8}(1-\lambda)\left({ }^{1}\right)
$$

ove $\lambda$ è una costante, $0<\lambda<1$.

Sia u una funzione di classe $C^{(2)}$ in $\Omega, C^{(0)}$ in $\bar{\Omega}$, soluzione dell'equazione

$$
\sum_{i, j}^{1, m} a_{i j} u_{i j}+\sum_{i=1}^{m-1} b_{i} u_{i}+\frac{b_{m}}{x_{m}}+c u-f=0 \quad \text { in } \Omega
$$

e verificante la (13). Esiste una costante $\tau$, dipendente soltanto da $\alpha, \lambda, K$ ed $\Omega$ per la quale sussiste la (18).

Proviamo che i teoremi II e III sono conseguenza del teorema I. Per il teorema II basta osservare che se $F$ è dato dal primo membro della (17), dalle (7), (8), (9), (10) seguono, per $M$ opportuno, le (15), (16). Per il teorema III sussiste una analoga osservazione ed inoltre dalle ipotesi relative a $\partial \Omega$ segue $\Omega \in H_{\varepsilon, \delta_{\varepsilon}}$ con $\varepsilon$ arbitrario in $\left(C, \frac{\pi}{2}\right)$, come è già stato notato al n. 1 .

4. Dimostrazione. - Articoliamo la dimostrazione del teorema I in quattro punti.

(1) A questa limitacione può essere sostituita l'ipotesi meno restrittiva

$$
b_{m}<\alpha \alpha(1-\lambda)
$$

con $\sigma$ costante minore di 1 . In questo easo $\tau$ dipende anche da $\sigma$. 
318 C. Puccr: Regolarità alla frontiora di soluzioni di equazioni ellittiche

4, a) Linearizzazione del problema. - Indicando con $x^{0}$ un punto di $\partial \Omega$ e posto

$$
\begin{gathered}
v(x)=u(x)-u\left(x^{0}\right), u^{0}=u\left(x^{0}\right), \\
A_{i j}(x)=\int_{0}^{1} \frac{\partial}{\partial u_{i j}} F\left[x, \tau u+(1-\tau) u^{0}, \tau u_{i}, \tau u_{i j}\right] d \tau, \\
A_{i}(x)=\int_{0}^{1} \frac{\partial}{\partial u_{i}} F\left[x, \tau u+(1-\tau) u^{0}, \tau u_{i}, \tau u_{i j}\right] d \tau, \\
A(x)=\int_{0}^{1} \frac{\partial}{\partial u} F\left[x, \tau u+(1-\tau) u^{0}, \tau u_{i}, \tau u_{i j}\right] d \tau,
\end{gathered}
$$

essendo

$$
F\left(x, u, u_{i}, u_{i j}\right)-F\left(x, u^{0}, 0,0\right)=\int_{0}^{1} \frac{d}{d \tau} F\left[x, \tau u+(1-\tau) u^{0}, \tau u_{i} \tau u_{i j}\right] d \tau
$$

si ha

$$
L v \equiv \sum_{i, j}^{1, m} A_{i j} v_{i j}+\sum_{i=1}^{m} A_{i} v_{i}+A v=-F\left(x, u^{0}, 0,0\right)
$$

$A_{i j}, A_{i}, A$ sono funzioni di $x$ definite in $\Omega$ e dalle $(7),(8),(9),(10)$ segue

$$
\alpha|\mu|^{2} \leq \sum_{i, j}^{i, m} A_{i j}(x) \mu_{i} \mu_{j} \leq|\mu|^{2} \quad \text { per } x \in \Omega
$$

$$
\begin{array}{rr}
d(x) n^{0} \times\left(A_{1}(x), \ldots, A_{m}^{\mathbb{w}}(x)\right)<\frac{\alpha}{8}(1-\lambda) & \text { per } x \in T\left(x^{0}, \frac{1}{2}\right), \\
d^{2}(x) \sum_{i=1}^{m} A_{i}^{2}(x) \leq K^{2}, d^{2-\lambda}(x) \quad A(x) \leq K & \text { per } x \in \Omega .
\end{array}
$$

Abbiamo già osservato che le ipotesi su $F$ sono invarianti rispetto a trasformazioni ortonormali delle coordinate e quindi sono pure invarianti le (24): (25), (26). Possiamo quindi supporre, per semplificare le notazioni, che il punto $x^{0}$ di $\partial \Omega$ fissato sia l'origine delle coordinate e che l'asse $x_{m}$ 
C. PUCCI : Regolarità alla frontiera di soluzioni di equazioni ellittiche 319

coincida in direzione e verso col versore $n\left(x^{\circ}\right)$. In questo caso la (25) assume l'aspetto

$$
A_{m}(x) d(x)<\frac{\alpha}{8}(1-\lambda), \quad \text { per } \quad x \in T\left(x^{0}, \frac{1}{2}\right)
$$

\section{4,b) Una funzione ausiliaria. - Poniamo}

$$
\begin{gathered}
\mathfrak{\vartheta}^{2}=\max \left[\frac{16 K}{(1-\lambda) \alpha}, \frac{4 m}{(1-\lambda) \alpha}\right], \quad \Re>0, \\
w\left(x, x^{0}\right)=\left[\sum_{i=1}^{m-1} x_{1}^{2}+\vartheta^{2} x_{m}^{2}\right]^{\frac{\lambda}{2}}=p^{2} .
\end{gathered}
$$

Segue

$$
\begin{aligned}
& w_{i}=\lambda p^{\lambda-2} x_{i} \text { per } i<m, \quad w_{m}=\lambda p^{\lambda-2 q^{2} x_{m}}, \\
& w_{i j}=-\lambda(2-\lambda) \rho^{\lambda-4} x_{i} x_{j}+\lambda \rho^{\lambda-2 \delta_{i j}} \quad \text { per } \quad i, j<m, \\
& w_{i m}=-\lambda(2-\lambda) p^{\lambda-4} x_{i} \vartheta^{2} x_{m} \quad \text { per } \mathrm{i}<m \\
& w_{m m}=-\lambda(2-\lambda) \rho^{\lambda-4} \vartheta^{4} x_{m}^{2}+\lambda \rho^{\lambda-2 q^{2}} \\
& L w=-\lambda \rho^{\lambda-2}\left\{(2-\lambda) \sum_{i, j}^{1, m-1} A_{i j} \frac{x_{i} x_{j}}{\rho^{2}}+2(2-\lambda) \sum_{i=1}^{m-1} A_{i m} \vartheta^{2} \frac{x_{i} x_{m}}{\rho^{2}}+\right. \\
& \left.+(2-\lambda) A_{m m} \vartheta^{4} \frac{x_{m}^{2}}{\rho^{2}}-\sum_{i=1}^{m-1} A_{i i}-A_{m m} \vartheta^{2}-\sum_{i=1}^{m-1} A_{i} x_{i}-A_{m} \vartheta^{2} x_{m}\right\}+A w .
\end{aligned}
$$

Siccome $H_{\beta, \delta} \subset H_{\beta, \sigma}$ se $\delta>\sigma$, senza alterare la generalità della dimostrazione si può supporre $\Omega \in H_{\beta}$, s con

$$
\delta<\left[\frac{(1-\lambda) \lambda \alpha}{64 K}\right]^{\frac{1}{\lambda}}
$$

Si ha

$$
\rho \leq \vartheta\left|x-x^{0}\right|, \quad d(x) \geq \frac{\left|x-x^{0}\right|}{2} \quad \text { per } x \in T\left(x^{0}, \frac{1}{2}\right)
$$

e quindi per le (26), (31) 


$$
A w<\frac{1-\lambda}{16} \alpha \lambda \vartheta^{2} p^{\lambda-2} \quad \quad \text { per } x \in T\left(x^{0}, \frac{1}{2}\right)
$$

Si ha per la (26)

$$
\left|\sum_{i=1}^{m-1} A_{i} x_{i}\right| \leq\left|x-x^{0}\right|\left[\sum_{i=1}^{m-1} A_{i}^{2}\right]^{\frac{1}{2}} \leq 2 K \quad \text { per } \quad x \in T\left(x^{0}, \frac{1}{2}\right),
$$

e quindi per la (28)

$$
\left|\sum_{i=1}^{m-1} A_{i} x_{i}\right| \leq \frac{1-\lambda}{8} \alpha \vartheta^{2} \quad \text { per } x \in T\left(x^{0}, \frac{1}{2}\right)
$$

Siccome

$$
x_{m} \leq\left|x-x^{0}\right| \leq 2 d(x) \quad \text { per } x \in T\left(x^{0}, \frac{1}{2}\right),
$$

dalle (27) segue

$$
A_{m} \vartheta^{2} x_{m}<\frac{1-\lambda}{4} \alpha \vartheta^{2} \quad \text { per } x \in T\left(x^{0}, \frac{1}{2}\right)
$$

Esiste una costante $\gamma, \gamma=\gamma(\alpha, \lambda)$ dipendente solamente da $\alpha$ e $\lambda$, tale che se $x \in \Omega$ e l'angolo $\eta$ formato fra il vettore $x-x^{0} \Theta$ l'asse $x_{m}$ è minore di $\gamma$ risulta

$$
\begin{gathered}
(2-\lambda)\left[\sum_{i, j}^{1,} A_{i j} \frac{x_{i} x_{j}}{\rho^{2}}+2 \sum_{i=1}^{m-1} A_{i m} \vartheta^{2} \frac{x_{i} x_{m}}{\rho^{2}}+A_{m m} \vartheta^{\vartheta^{2}} \frac{x_{m}^{2}}{\rho^{2}}\right]- \\
-\sum_{i=1}^{m-1} A_{i i}-A_{m m} \vartheta^{2}>\frac{1-\lambda}{2} \Re^{2} \alpha .
\end{gathered}
$$

Osserviamo infatti che per la (24) il primo membro è maggiore di

$$
-4 \sum_{i=1}^{m-1} \Re^{2} \frac{\left|x_{i} x_{m}\right|}{\rho^{2}}-\vartheta^{2}\left(1-\frac{\vartheta^{2} x_{m}^{2}}{\rho^{2}}\right)+(1-\lambda) A_{m m} \vartheta^{2}-\sum_{i=1}^{m-1} A_{i i}
$$

ed essendo

$$
\left|x_{i}\right| \leq p \operatorname{sen} \eta, \quad 1-\frac{\eta^{2} x_{m}^{2}}{\rho^{2}} \leq \operatorname{sen}^{2} \eta \leq \operatorname{sen} \eta
$$

il primo membro della (35) è maggiore di

$$
(1-\lambda) A_{m m^{9^{2}}}-\sum_{i=1}^{m-1} A_{i i}-8 m \vartheta^{2} \operatorname{sen} \eta
$$
e questa quantità per le $(24),(28)$ è maggiore di $\frac{3}{4}\left(1-\lambda \mid \vartheta^{2} \alpha\right.$ quando $\eta$ è uguale
a zero. 
Supponiamo da qui in avanti $\boldsymbol{Q} \in H_{\beta, \delta}$ con $\beta \leq \frac{1}{2} \gamma(\alpha, \lambda)$; per le (3), (4) esiste un $t$, maggiore di $\frac{1}{2}$, tale che $\gamma_{t} \leq \gamma(\alpha, \lambda)$; la (35) sussiste quindi in $T\left(x^{0}, t\right)$. Siccome

$$
T\left(x^{0}, t\right) \subset T\left(x^{0}, \frac{1}{2}\right)
$$

le (32), (33), (34), (35) sussistono simultaneamente in $T\left(x^{0}, t\right)$; da esse e dalla (30) segue, nell' ipotesi $\beta \leq \frac{1}{2} \gamma(\alpha, \lambda)$,

$$
L w \leq-\frac{1-\lambda}{16} \lambda \alpha \vartheta^{2} p^{\lambda-2} \quad \text { per } x \in T\left(x^{0}, t\right)
$$

Le costanti $\vartheta, t$ e $\gamma(\alpha, \lambda)$ sono state fissate indipendentemente dal punto $x^{0}$ considerato e pertanto $w\left(x, x^{0}\right)$ soddisfa alla (36), qualunque sia $x^{0}$ su $\partial Q$.

4,c) Una prima limitazione. Poniamo

$$
H_{1}=\sup _{\substack{x, \in \in a \\ x \in T\left(x^{0}, t\right)}} \frac{u(x)-u\left(x^{0}\right)}{w\left(x, x^{0}\right)}
$$

Vogliamo provare che se $\Omega \in H_{\beta, \delta}$, con $0<\beta<\beta_{0}(\alpha, \lambda, k, m)$, si ha

$$
H_{1} \leq \frac{64}{(1-\bar{\lambda}) \lambda \alpha} \mathfrak{F}+\frac{2}{\delta^{\lambda}} \max _{x \in 0}|u(x)|+\tau_{1} h,
$$

con $\tau_{1}$, costante positiva dipendente da $\alpha, \lambda, K, m$ soltanto.

Supponiamo dapprima

$$
H_{2}=\sup _{\substack{x^{0} \in \partial \varrho \\ x \in \partial T\left(x^{0}, \bar{t}\right)}} \frac{u(x)-u\left(x^{0}\right)}{w\left(x, x^{0}\right)}<H_{1} ;
$$

supposto $H_{1}>0$ (in caso contrario la (38) è soddisfatta), esiste un $H_{3}$ tale che

$$
H_{2}<H_{3}<H_{1}, \quad H_{3}>\frac{1}{2} H_{1}
$$

si ha

$$
g\left(x, x^{0}\right)=H_{3} \quad w\left(x, x^{0}\right)-\left[u(x)-u\left(x^{0}\right)\right] \geq 0 \text { per } x \in \partial T\left(x^{0}, t\right),
$$

e per le (21), (25), (36) si ha

$$
L g \leq-H_{3} \frac{1-\lambda}{16} \lambda \alpha \vartheta^{2} p^{\lambda-2}+F\left(x, u^{0}, 0,0\right) \quad \text { per } \quad x \in T\left(x^{0} t\right) .
$$


Dalle (37), (39), (40) segue che vi è un punto $x^{0} \in \partial Q$ e un $x \in T\left(x^{0}, t\right)$ tali che $g\left(x, x^{0}\right)<0$. Pertanto per la $(41)$ e il principio di massimo non può essere $L g \leq 0$ in $T\left(x^{0}, t\right)$ e quindi

$$
\sup _{x \in T\left(x^{0}, t\right)}\left[-H_{3} \frac{1-\lambda}{16} \lambda \alpha \vartheta^{2} \rho^{\lambda-2}+F\left(x, u^{0}, 0,0\right)\right]>0 \text {. }
$$

Siccome

$$
\begin{gathered}
\frac{\rho}{\grave{9}} \leq\left|x-x^{0}\right| \leq \frac{d(x)}{t} \quad \text { in } T\left(x^{0}, t\right), \\
F\left(x, u^{0}, 0,0\right)=F(x, 0,0,0)+u^{0} F_{u}\left(x, \sigma u^{0}, 0,0\right) \quad \text { con } 0<\sigma<1,
\end{gathered}
$$

si ottiene

$$
H_{\mathrm{z}}<\frac{16}{(1-\lambda) \lambda \alpha t^{9 \lambda}} \sup _{x \in T\left(x^{0}, t\right)} d^{2-\lambda}(x)\left|F(x, 0,0,0)+u^{0} F_{u}\left(x, \sigma u^{0}, 0,0\right)\right|
$$

siccome $H_{1}<2 H_{3}$, per le $(9),(11)$, essendo $\vartheta>1, t>\frac{1}{2}$, risulta

$$
H_{1}<\frac{64}{(1-\lambda) \lambda \alpha}\left(\mathbb{F}+K \max _{x \in 0}|u(x)|\right)
$$

e quindi la (38) sussiste nell' ipotesi (39).

Consideriamo ora il easo che la (39) non sussista. Se

$$
H_{1}=\sup _{\substack{x^{0} \in \partial a \\ \mid x-x_{0} 0 .=\delta \\ x \in \partial T\left(x^{0}, t\right)}} \frac{u(x)-u\left(x^{0}\right)}{w\left(x, x^{0}\right)}
$$

risulta

$$
H_{1} \leq \frac{2}{\delta^{2}} \max _{x \in 0}|u(x)|
$$

e quindi anche in tale caso è verificata la (38).

Se non sussiste la (39) nè la (42) risulta

$$
H_{1}=\sup _{\substack{x x^{0} \in \partial 0 \\\left|x-x^{0}\right|<\delta \\ x \in \partial T\left(x^{0}, t\right)}} \frac{u(x)-u\left(x^{0}\right)}{w\left(x, x^{0}\right)}
$$

Sia $x \in \partial T\left(x^{0}, t\right)$ con $\left|x-x^{0}\right|<\delta$; ne segue $t\left|x-x^{0}\right|=d(x)$. Indichiamo con $x^{\prime}$ uno dei punti di $\partial \mathrm{Q}$ più prossimi ad $x:\left|x-x^{\prime}\right|=d(x)$. 
Si ha quindi $x \in T\left(x^{\prime}, t\right)$ e, per la (37),

(44)

$$
\frac{u(x)-u\left(x^{\prime}\right)}{w\left(x, x^{\prime}\right)} \leq H_{1}
$$

Notiamo che

$$
\begin{gathered}
\frac{u(x)-u\left(x^{0}\right)}{w\left(x, x^{0}\right)}=\frac{u(x)-u\left(x^{\prime}\right)}{w\left(x, x^{\prime}\right)} \frac{w\left(x, x^{\prime}\right)}{w\left(x, x^{0}\right)}+\frac{u\left(x^{\prime}\right)-u\left(x^{0}\right)}{\left|x^{\prime}-x^{0}\right|^{\lambda}} \frac{\left|x^{\prime}-x^{0}\right|^{\lambda}}{w\left(x, x^{0}\right)} \\
\left|x^{\prime}-x^{0}\right| \leq\left|x-x^{0}\right|+\left|x-x^{\prime}\right|<2\left|x-x^{0}\right|, \quad\left|x^{\prime}-x^{0}\right|^{\lambda}<2^{\lambda} w\left(x, x^{0}\right) \\
w\left(x, x^{\prime}\right) \leq \vartheta^{\lambda}\left|x-x^{\prime}\right|^{\lambda}=\vartheta^{\lambda} t^{\lambda}\left|x-x^{0}\right|^{\lambda} \\
w\left(x, x^{0}\right)=\left[\operatorname{sen}^{2} \eta+\vartheta^{2} \cos ^{2} \eta\right]^{\frac{\lambda}{2}}\left|x-x^{0}\right|^{\lambda}
\end{gathered}
$$

ove $\eta$ denota l'angolo formato fra l'asse $x_{m}$ e il vettore $x-x^{0}$. Si ha quindi per le (13), (44)

$$
\frac{u(x)-u\left(x^{0}\right)}{w\left(x, x^{0}\right)} \leq H_{1}\left\{\frac{t^{2}}{g^{-2} \operatorname{sen}^{2} \eta+\cos ^{2} \eta}\right\}^{\frac{\lambda}{2}}+2 h, \text { per } x \in T\left(x^{0}, t\right),\left|x-x^{0}\right|<\delta .
$$

Siccome la funzione $\vartheta^{-2} \operatorname{sen}^{2} \gamma_{i}+\cos ^{2} \eta$ è decrescente per $\eta$ variabile in $0, \frac{\pi}{2}$, essendo $\eta \leq \gamma_{t}$ per la definizione di $\gamma_{t}$, si ha

$$
\vartheta^{-2} \operatorname{sen}^{2} \eta+\cos ^{2} \eta \geq \vartheta^{-2} \operatorname{sen}^{2} \gamma_{t}+\cos ^{2} \gamma_{t}
$$

Per la (5) si ottiene

$$
\frac{t^{2}}{\vartheta^{-2} \operatorname{sen}^{2} \eta+\cos ^{2} \eta} \leq \frac{t^{2}}{\vartheta^{-2} \operatorname{sen}^{2}(\beta+\arccos t)+\cos ^{2}(\beta+\arccos t)} .
$$

Se poniamo $\beta=0$ nel secondo membro otteniamo

$$
\frac{t^{2}}{\mathfrak{t}^{-2}\left(1-t^{2}\right)+t^{2}}<1
$$

pertanto esistono due costanti positive $\beta_{1}$ e $\sigma$ dipendenti solamente da $\vartheta$ e $t$ e quindi da $\alpha, \lambda, m, k$ tali che

Dalla (45) segue

$$
\frac{t^{2}}{\eta^{-2} \operatorname{sen}^{2} \eta+\cos ^{2} \eta} \leq \sigma<1 \quad \text { per } \beta \leq \beta_{1} .
$$

$$
H_{1} \leq \frac{2}{1-\sigma} h
$$


e quindi la (38) nell'ipotesi $\beta \leq \beta_{1}(\alpha, \lambda, k, m)$.

La (38) resta quindi provata se $\Omega \in H_{\beta, \delta}$ con $0<\beta \leq \beta_{0}$ ove $\beta_{0}$ è il minimo $\operatorname{fra} \frac{1}{2} \gamma(\alpha, \lambda) \mathrm{e}^{\varepsilon} \beta_{1}(\alpha, \lambda, k, m)$.

4,d) Conclusione. Poniamo

$$
H=\sup _{\substack{x^{0} \in \delta^{n} \\ x \in \Omega}} \frac{u(x)-u\left(x^{0}\right)}{\left|x-x^{0}\right|^{\lambda}} .
$$

Siccome $\vartheta\left|x-x^{0}\right|>w\left(x, x^{0}\right)$ si ha

$$
\frac{u(x)-u\left(x^{0}\right)}{\left|x-x^{0}\right|^{2}}<\Re^{\lambda} H_{1} \quad \text { per } x^{0} \in \partial \Omega, x \in T\left(x^{0}, t\right)
$$

Inoltre si ha

$$
\frac{u(x)-u\left(x^{0}\right)}{\left|x-x^{0}\right|^{\lambda}} \leq \frac{2}{\delta^{\lambda}} \max _{x \in \Omega}|u(x)| \quad \text { per } x^{0} \in \partial Q, x \in Q,\left|x-x^{0}\right| \geq \delta
$$

Se $x$ appartiene ad $\Omega$ ma non a $T\left(x^{0}, t\right)$ e $\left|x-x^{0}\right|<\delta$ allora deve essere $t\left|x-x^{0}\right|>d(x)$; in questo caso, indicato con $x^{\prime}$ uno dei punti di $\partial \mathbf{Q}$ più prossimi ad $x$; cioè $\left|x-x^{\prime}\right|=d(x)$, siccome

$$
\begin{gathered}
\frac{u(x)-u\left(x^{0}\right)}{\left|x-x^{0}\right|^{\lambda}}=\frac{u(x)-u\left(x^{\prime}\right)}{\left|x-x^{\prime}\right|^{\lambda}} \frac{\left|x-x^{\prime}\right|^{\lambda}}{\left|x-x^{0}\right|^{\lambda}}+\frac{u\left(x^{\prime}\right)-u\left(x^{0}\right)}{\left|x^{\prime}-x^{0}\right|^{\lambda}} \frac{\left|x^{\prime}-x^{0}\right|^{\lambda}}{\left|x-x^{0}\right|^{\lambda}}, \\
\left|x^{\prime}-x^{0}\right|<\left|x-x^{0}\right|+\left|x-x^{\prime}\right|=(1+t)\left|x-x^{0}\right|
\end{gathered}
$$

risulta

Se

dalla (49) segue

$$
\frac{u(x)-u\left(x^{0}\right)}{\left|x-x^{0}\right|^{2}} \leq H t^{\lambda}+h(1+t)^{\lambda} \quad \text { per } x^{0} \in \partial \Omega, x \in \Omega, t\left|x-x^{0}\right|>d(x)
$$

$$
H=\sup _{\substack{x \\ x \in \partial \Omega, x \in \Omega \\ t\left|x \rightarrow x^{0}\right|>d(x)}} \frac{u(x)-u\left(x^{0}\right)}{\left|x-x^{0}\right|^{\lambda}}
$$

$$
H \leq \frac{(1+t)^{\lambda}}{1-t^{2}} h
$$

Se la (50) non sussiste dalle (47), (48) segue

$$
H \leq \Re^{\lambda} H_{1}+\frac{2}{\delta^{\lambda}} \max _{x \in \bar{\Omega}}|u(x)|
$$


Si è quindi costante $\tau$, dipendente solamente da $\alpha, \lambda, K, m, \delta$, tale che

$$
u(x)-u\left(x^{0}\right) \leq \tau\left(h+\mathscr{F}+\max _{x \in \Omega}|u(x)|\right)\left|x-x^{0}\right|^{\lambda} \quad \text { per } x^{0} \in \partial \Omega, x \in \mathbf{Q} .
$$

In eguale modo si prova la limitazione inferiore per $u(x)-u\left(x^{0}\right)$ e per. tanto il teorema I è dimostrato.

5. Ossenvazioni. - Nel teorema I si suppone $\mathbf{Q} \in H_{\beta, \delta}$ con $\beta$ minore una costante opportuna $\beta(\alpha, \lambda, k, m)$. Questa ipotesi è essenziale vale a dire ferme rimanendo le ipotesi su $F$ ed $u$, e l'ipotesi della appartenenza di $\Omega$ a qualche $H_{\beta, \delta}$, la limitazione (14) paò non sussistere se $\beta$ non è sufficientemente piccolo. Mostriamo ciò con un esempio.

Sia $\beta$ una costante positiva minore di $\frac{\pi}{2}$ e sia $\Omega$ l'insieme dei punti del piano $x \equiv\left(x_{1}, x_{2}\right)$ tali che $|x|<1$ e $0<$ argomento di $x<\pi+2 \beta$. Risulta $\Omega \in H_{\beta, \delta} \operatorname{con} \delta$ positivo arbitrario. Sia $\lambda$ una costante, $0<\lambda<1$. Sia $u$ una funzione di classe $C^{(2)}$ in $\Omega, C^{(0)}$ in $\bar{\Omega}$, con

$$
u_{x_{1} x_{1}}+u_{x_{2} x_{2}}=0 \quad \text { in } \Omega \text {. }
$$

$$
\left|u(x)-u\left(x^{0}\right)\right| \leq 2\left|x-x^{0}\right|^{\lambda} \quad \text { per } x, x^{0} \in \partial \Omega \text {. }
$$

Posto $F\left(x, u, u_{i}, u_{i j}\right)=u_{11}+u_{22}$, le ipotesi del teorema I sono verificate eccettuata quella riguardante la sufficiente piccolezza di $\beta$. Proviamo che se è

$$
\beta>\pi \frac{1-\lambda}{2 \lambda}
$$

Ia limitazione (14) può non sussistere. Basta considerare la funzione

$$
u(x)=|x|^{\frac{\pi}{2 \beta+\pi}} \operatorname{sen}\left[\frac{\pi}{2 \beta+\pi} \arg \cdot x\right]
$$

La $u$ è armonica in $Q$, ̀̀ nulla sulle semirette arg. $x=0$, e arg. $x=\pi+2 \beta$ e per questo si verifica la $(56)$.

Si ha

$$
u(x)-u(0)=|x|^{\frac{\pi}{2 \beta+\pi}} \quad \text { per arg. } x=\beta+\frac{\pi}{2},
$$

pertanto la (14) non sussiste se $\beta$ non soddisfa alla limitazione (57).

$L$ 'esempio considerato mostra che $\beta$ non è indipendente da $\lambda$. Con un analogo esempio si può osservare la necessaria dipendenza di $\beta$ anche da $\alpha$. 
Basta considerare la funzione

$$
v\left(x_{1}, x_{2}\right)=u\left(x_{1}, \sqrt{\alpha} x_{2}\right)
$$

ove $u$ è dato dalla $(58)$ ed $\alpha$ ¿ una costante positiva minore di 1 . La $v$ verifica l'equazione

$$
\alpha v_{x_{1} x_{1}}+v_{x_{2} x_{2}}=0
$$

nell' insieme $Q$ intersezione di un ellisse e di un angolo:

$$
\Omega \equiv\left\{x_{1}, x_{2} ; x_{1}^{2}+\alpha x_{2}^{2}<1,0<\arg x<\pi+2 \beta^{*}\right\} \quad \text { con } 2 \beta^{*}=\operatorname{arcsen} \frac{\operatorname{sen} 2 \beta}{\sqrt{\alpha}}
$$

$\mathbf{Q}$ appartiene ad $H_{\beta^{*}, \delta}$ con $\delta$ positivo arbitrario,

$$
v=0 \quad \text { per } \arg x=0 \quad \text { oppure } \arg x=\pi+2 \beta^{*}
$$

e. pertanto si verifica

$$
\left|v(x)-v\left(x^{0}\right)\right| \leq 2\left|x-x^{0}\right|^{2} \quad \text { per } x, x^{0} \in \partial \Omega,(0<\lambda<1) .
$$

Affinchè $v(x)-v(0)$ si possa maggiorare con $|x|^{\lambda}$ occorre che

$$
\beta^{*} \leq \frac{1}{2} \operatorname{arcsen}\left[\frac{1}{\sqrt{\alpha}} \operatorname{sen} \frac{1-\lambda}{\lambda} \pi\right]
$$

Una questione interessante che mi è stata posta da AgMoN è la seguente: supposto che $\Omega$ appartenga ad un qualche $H_{\beta, \delta}$ e che $F$ ed $u$ soddisfino alle ipotesi del teorema I (la (13) supposta verificata comunque si fissi $\lambda$ con $0<\lambda \leq \lambda_{0}<1$ ), esiste un $\lambda$ positivo e sufficientemente piccolo per il quale sussiste la (14)?

Si tratterebbe quindi di determinare delle condizioni per $\lambda$ invece che per $\beta$. Ritengo che la risposta sia affermativa ma non mi è riuscito proviario; probabilmente occorre una funzione ausiliaria diversa da $w\left(x, x^{0}\right)$.

Un'altra questione interessante che mi è stata posta da STAMPacchIa è la seguente: sia $x^{0}$ un punto di $\partial Q$, ed indichiamo con $\varphi$ una funzione continua su $\partial \Omega$ con

$$
\left|\varphi(x)-\varphi\left(x^{0}\right)\right| \leq h\left|x-x^{0}\right|^{2} \quad \text { per } x \in \partial \Omega,(0<\lambda<1),
$$

e con $u$ la corrispondente soluzione del problema di DrrroHLeT

$$
\Delta u=0 \quad \text { in } \Omega, \quad u=\varphi \quad \text { su } \partial \Omega
$$


$x^{0}$ si dice « $\lambda$-regolare per $\Omega \gg$ se comunque fissata la funzione $\varphi$ verificante le ipotesi precedenti esiste una costante $H$, dipendente solo da $h, \lambda$ ed $\Omega$ tale che

$$
\left|u(x)-u\left(x^{0}\right)\right| \leq H\left|x-x^{0}\right|^{\lambda} \quad \text { per } x \in \Omega
$$

quali sono le condizioni necessarie e sufficienti relative a $\partial \Omega$ affinchè $x^{0}$ sia $\lambda$-regolare per $\Omega ?$ Naturalmente si deve trattare di condizioni dipendenti da $\lambda$ come segue anche dal primo esempio considerato, e quindi diverse da quelle introdotte da Wrener. Osserviamo anche che è falsa la seguente estensione alla $\lambda$-regolarità dei teoremi di Herné, LimTman, STAMPaOCHIA, Whinberger già ricordati: «un punto $x^{0}$ di $\partial \Omega$ è $\lambda$-regolare per un operatore ellittico sufficientemente liscio se e solo se è $\lambda$-regolare per l'operatore di LAPrado ». Il secondo esempio infatti mostra che nelle condiaioni di $\lambda$-regolarità deve anche intervenire la costante di elliticità dell'operatore considerato.

\section{BIBLIOGRAFIA}

[1] S. Agmon - A. Dovalis - L. Nirenberg, Estimates near the boundary for solutions of elliptic partial differential equations satisfying general boundary conditions, I *Comm. pure appl. math."12, 1959, pp. 623-727.

[2] L. Bers - L. NIRENBERG, On linear and non-linear elliptic boundary value problems in the plane, «Convegno Int. Eq. a derivate parz.» Trieste 1954.

[3] R. CACCIOPPOLI, Sulle equasioni ellittiche a derivate paraiali con $n$ variabili indipen. denti, "Rend. Acc. Lincei * 19, 1934, 83-89.

[4] E. De Glorgr, Sulla differenziabilità e l'analiticità delle estremali degli integrali mul. tipli regolari, «Mem. Acc. Sc. Torino» III, Parte I, pp. 205 -43 (1957).

[5] R. FINN - J. SERRIN, On the Hölder continuty of quasi-conformal and elliptic mappings, «Trans. Amer. Math. Soc.» 89, (1958) pp. 1-15.

[6] R. HERni, Recherches axionatiques sur la thérie des fonctions surharmoniques et du potential, «Ann. Ist. Fourier, Grenoble 12, (1962), pp. 415-5̄7.

17] O. KeLrog, On the derivatives of harmonic functions on the boundary, "Trans. Amer. Math. Soc.» 33 (1931) pp. 486.510.

[8] H. Lebesgue, Sur des cas d'impossibilité au probleme de Dirichlet, "Comptes Rendus de Ia Soc. Math. dé France» (1913).

[9] Litrman, STAMPaccma, WeInberger, Regular points for elliptic equations with discontinuous coefficients, «Annali Scuola Norm. Pisa》 IIT, 17, (1963) pp. 45.79.

[10] C. Miranda, Equazioni alle derivate parziali ai tipo ellitico, «Ergeb. der Math. Sprin. ger" Verlag Berlin (1955).

[11] J. Moser, On Harnack theorem for elliptic differential equations, “Comm. Pure Applied Math.» 14, pp. 577-591, (1961).

[12] J. NASH, Continuity of the solutions of parabolic and elliptic equations, "Amer. J. Math. * 80, pp. 981.054, (1959).

[13] L. NIRENBERG, On non-linear elliptic partial differential equations and Hölder continuity, ¿Comm. pure appl. math. 6, (1953), pp. 103-150̄. 
328 C. PuccI: Regolarità alla frontiera di soluzioni di equazioni ellittiche

[14] O. OLEñK, Sul problema di Dirichlet per le equazioni di tipo ellittico (in russo) Math. Sbr. N. S. 24, 66 (1949), pp. 3-14.

[15] C. Puccr, Sulle funzioni barriera, "Le Matematiche» 1963.

[16] - - Lectures on the Cauchy Problem and on Second Order Elliptic Equations "National Science Foundation, Summer Institute in Applied Math.» Rice University 1963.

[17] W. Püsches, Dier erste Randverttaufgabe der allgemeinen sebbstradjungierten elliptischen Differentialgleichung zweiter Ordnung fur beliebige Gebiete, «Math. Zeit» 34, (1931), pp." 535.5553 .

[18] J. Schauder, Über lineare elliptische Differentialgleichungen zweiter Ordnung, "Math. Z. 38 (1934) 2,7.282),

[19] G. TAUTZ, Zur Theorie der ersten Randwertanfgabe, "Math. Nach. » 2 (1949) pp. 279-303.

[20] IN. WIener, The Dirichlet problem, «J. Math. and Phys. 3, (1924), pp. 127.146. 